\title{
Correction: Global deletion of optineurin results in altered type I IFN signaling and abnormal bone remodeling in a model of Paget's disease
}

\author{
Sing-Wai Wong $\mathbb{D}$ - Bo-Wen Huang - Xiangxiang Hu • Eui Ho Kim • Joseph P. Kolb • Ricardo J. Padilla • Peng Xue • \\ Lufei Wang - Thomas H. Oguin III - Patricia A. Miguez - Henry C. Tseng • Ching-Chang Ko • Jennifer Martinez
}

Published online: 16 July 2020

(c) The Author(s), under exclusive licence to ADMC Associazione Differenziamento e Morte Cellulare 2020, corrected publication 2022

Correction to: Cell Death \& Differentiation https://doi.org/10.1038/s41418-019-0341-6

Following publication of this article, the authors noticed that there was an error in the legend for Fig. 3. The relevant and corrected section is provided below:

$(\mathrm{D}, \mathrm{G}) \mathrm{Optn}^{+/+}$and $\mathrm{Optn}^{-/-}$bone-marrow-derived precursors were cultured under osteoclastogenic conditions, and autophagic activity (d) and noncanonical NF- $\mathrm{KB}$ signaling (g) was assessed by western blot on days 0-3 post RANKL treatment from the sample protein lysates. In d, cells were probed for OPTN, LC3, p62, ATG5, ATG7, and $\beta$-actin. In $\mathbf{g}$, the western blots were reprobed for $\mathrm{p} 100 / \mathrm{p} 52$ with the same OPTN and $\beta$-actin bands shown for reference. e, f $\mathrm{Optn}^{+/+}$and $\mathrm{Optn}^{-1-}$ bone-marrow-derived precursors were cultured with RANKL for 0-60 min or 0-3 days, to assess the activation of canonical NF- $\kappa \mathrm{B}$ signaling by western blot. Cells were probed for OPTN (e, f), phos-p65 $(\mathbf{e}, \mathbf{f})$, Total p65 (f), Total IkB (e), and $\beta$-actin $(\mathbf{e}, \mathbf{f})$.

In addition, in the $100 \mu \mathrm{m}$ TRAP panel of Fig. $2 \mathrm{~b}$, an adjacent serial histologic section from the same sample was inadvertently used during image compilation. The correct image is provided below, the correct $100 \mu \mathrm{m}$ TRAP image is from the same histologic section as that used for the $1 \mathrm{~mm}$ TRAP image.

The authors apologise for these errors. 
Fig. 2

A

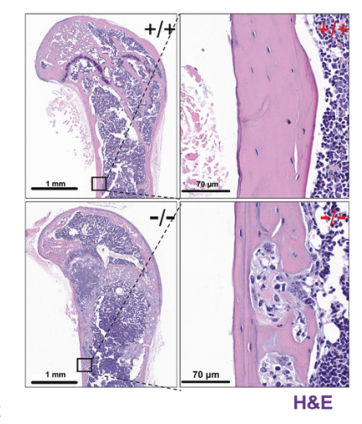

C

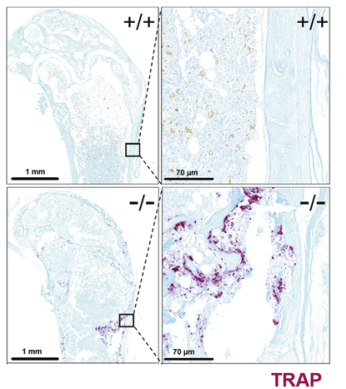

E

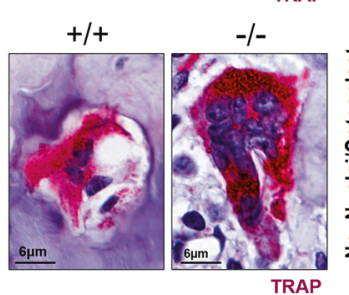

B

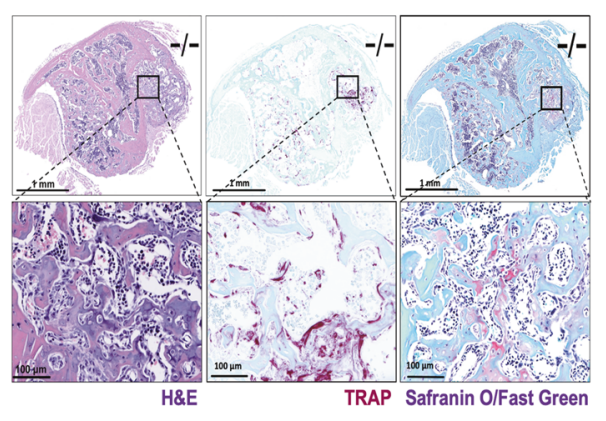

D
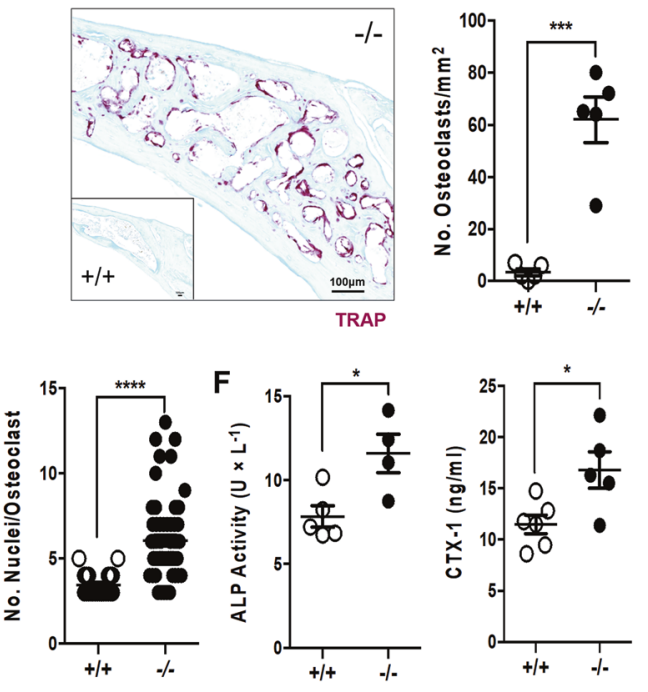

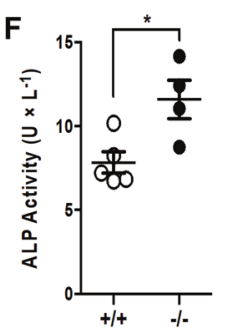

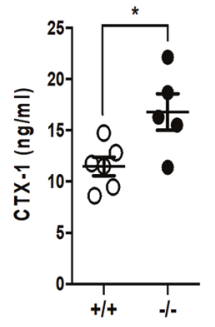

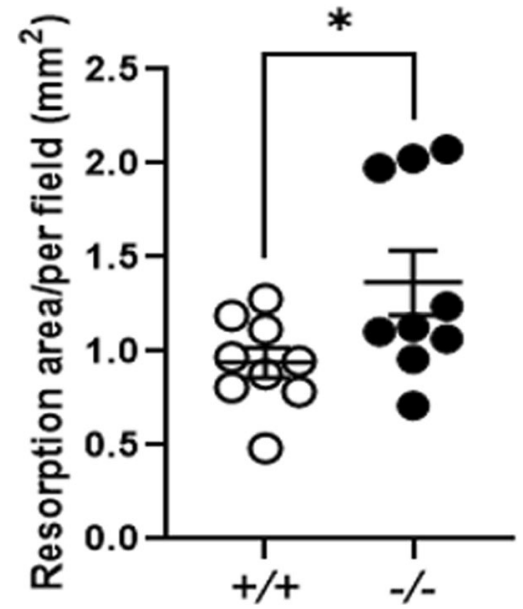

Fig. 3 